\title{
ECG Analysis using Continuous Wavelet Transform (CWT)
}

\author{
Apoorv Gautam ${ }^{1}$ and Maninder Kaur ${ }^{2}$ \\ ${ }^{I}$ (ECE Deptt., Doaba Institute of Engineering \& Technology, Kharar, Punjab INDIA,) \\ ${ }^{2}$ (ECE Deptt., Doaba Institute of Engineering \& Technology, Kharar, Punjab INDIA,)
}

\begin{abstract}
One of the important step in the ECG analysis is to accurately detect the different waves forming the entire ecg. The wavelet transformation is worth investigating in P- and T-wave recognition. The benefit of the wavelet based ECG approach is that $\mathrm{T}$-wave abnormalities can be assessed without the need for T-wave end point identification. The wavelet transformation is a new promising technique in non-invasive electro cardiology providing improved methods for late potential detection. In the presented paper work, The proposed paper work is aimed to analyse a given ecg by using wavelet transform and to identify the Arrhythmia. The present approach and results can be implemented in the ecg machines to give analysis report along with the ecg of the patient. The accurate and authentic analysis of the ecg in ecg machine is very much in demand today. Further, it is proposed to extract certain arrhythmia patterns from the ecg of different patients to enable an ecg machine to correlate the analysis based on the history.
\end{abstract}

\section{INTRODUCTION}

Electrocardiograms (ECGs) are signals that originate from the action of the human heart. The ECG is the graphical representation of the potential difference between two points on the body surface, versus time. Each heartbeat is a complex of distinct cardiological events, represented by distinct features in the ECG waveform. ECG recordings are examined by a physician who visually checks features of the signal and estimates the most important parameters of the signal. Using this expertise the physician judges the status of a patient. Therefore the recognition and analysis of the ECG signals is a very important task. The standard parameters of the ECG waveform are the $\mathrm{P}$ wave, the QRS complex and the $\mathrm{T}$ wave. But most of the information lies around the $\mathrm{R}$ peak. Additionally a small $\mathrm{U}$ wave (with an uncertain origin) is occasionally present.

\section{BRIEF LITERATURE SURVEY}

The earlier method of ECG signal analysis was based on time domain method. But this is not always sufficient to study all the features of ECG signals. So, the frequency representation of a signal is required. To accomplish this, FFT (Fast Fourier Transform) technique is applied [5]. But the unavoidable limitation of this FFT is that the technique failed to provide the information regarding the exact location of frequency components in time [3]. As the frequency content of the ECG Signal Analysis Using Wavelet Transforms ECG varies in time, the need for an accurate description of the ECG frequency contents according to their location in time is essential. This justifies the use of time frequency representation in quantitative electro cardiology. The immediate tool available for this purpose is the Short Term Fourier Transform (STFT) [1]. But the major draw-back of this STFT is that its time frequency precision is not optimal. Hence we opt a more suitable technique to overcome this drawback. Among the various time frequency transformations the wavelet transformation is found to be simple and more valuable [2]. The wavelet transformation is based on a set of analyzing wavelets allowing the decomposition of ECG signal in a set of coefficients [7]. Each analyzing wavelet has its own time duration, time location and frequency band. The wavelet coefficient resulting from the wavelet transformation corresponds to a measurement of the ECG components in this time segment and frequency band.

\section{ECG WAVE PATTERN}

In the normal sinus rhythm (normal state of the heart) the P$\mathrm{R}$ interval is in the range of 0.12 to 0.2 seconds. The QRS interval is from 0.04 to 0.12 seconds. The Q-T interval is less than 0.42 seconds and the normal rate of the heart is from 60 to 100 beats per minute. So, from the recorded shape of the ECG, we can say whether the heart activity is normal or abnormal. The normal value of heart beat lies in the range of 60 to 100 beats/minute. A slower rate than this is called bradycardia (Slow heart) and a higher rate is called tachycardia (Fast heart). If the cycles are not evenly spaced, an arrhythmia may be indicated. If the P-R interval is greater than 0.2 seconds, it may suggest blockage of the AV node. Below figure shows an ideal ecg waveform [9].

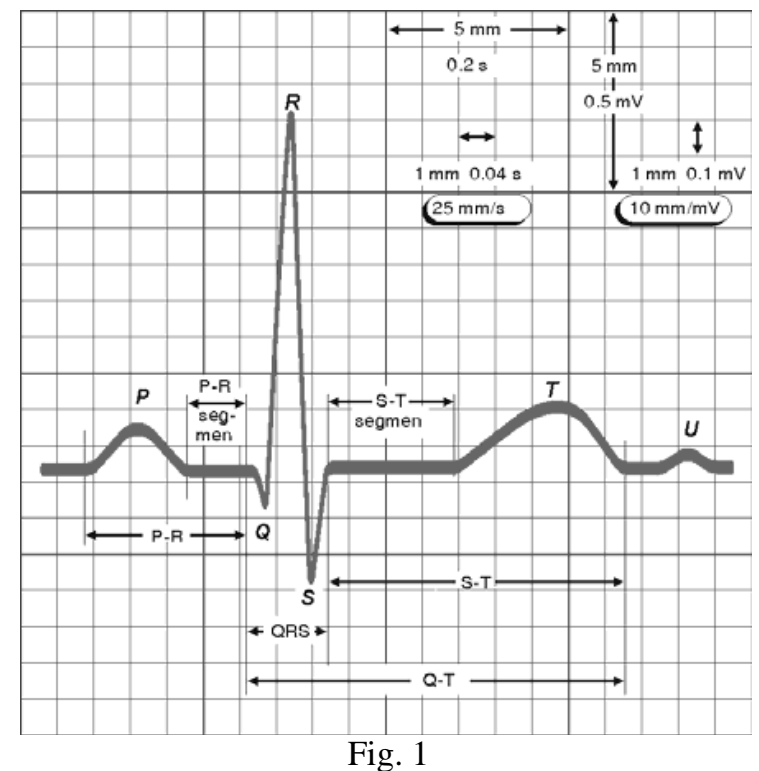

Fig. 1 
The cardiac cycle begins with the $\mathrm{P}$ wave, which corresponds to the period of atrial depolarization in the heart. This is followed by the QRS complex, which is usually the most relevant (recognizable) feature of an ECG waveform. The $\mathrm{T}$ wave follows the QRS complex and corresponds to the period of ventricular repolarization. The end point of the $\mathrm{T}$ wave represents the end of the cardiac cycle (presuming the absence of $U$ wave). The durations (time between the onset and offset) of particular parameters of the ECG (referred as an time interval) is of great importance since it provides a measure of the state of the heart and can show the presence of certain cardiological conditions.

\section{ARRHYTHMIA}

Disturbance in the regular rhythmic activity of the heart. Cardiac arrhythmia may be caused by irregular firing patterns from the SA node, abnormal activity from other parts of the heart. Below table presents deviations from the normal values of ECG features (segments and intervals) and respective arrhythmia type [9]. Normal, trachycardia and bradicardia ecgs are shown in below figures 2, 3 and 4 respectively.

\begin{tabular}{cll}
\hline S. No. & Name of abnormality & Characteristic features \\
\hline 1 & Dextrocardia & Inverted P-wave \\
2 & Tachycardia & R-R interval $<0.6 \mathrm{~s}$ \\
3 & Bradycardia & R-R interval $>1 \mathrm{~s}$ \\
4 & Hyperkalemia & Tall T-wave and absence of P-wave \\
5 & Myocardial ischaemia & Inverted T-wave \\
6 & Hypercalcaemia & QRS interval $<0.1 \mathrm{~s}$ \\
7 & Sinoatrial block & Complete drop out of a cardiac cycle \\
8 & Sudden cardiac death & Irregular ECG
\end{tabular}

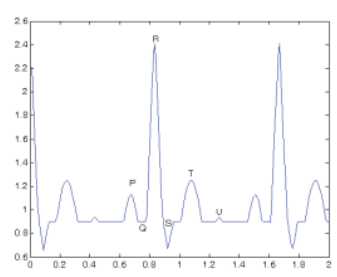

Fig. 2 Normal ECG

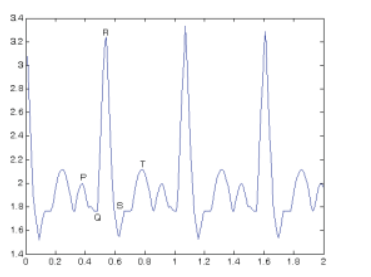

Fig. 3 Tachycardia

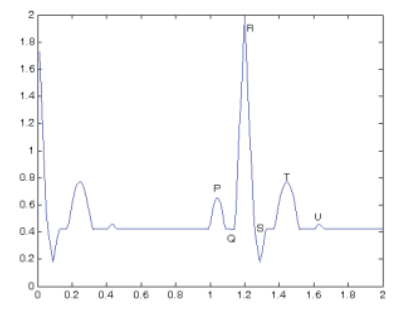

Fig. 4 Bradycardia

\section{WAVELET TRANSFORM}

The wavelet transform has emerged over recent years as the most favoured tool by researchers for analysing problematic signals across a wide variety of areas in science, engineering and medicine. Wavelets are mathematical functions that cut up data into different frequency components, and then study each component with a resolution matched to its scale. Key advantage of wavelet techniques is the variety of wavelet functions available, thus allowing the most appropriate to be chosen for the signal under investigation.

Wavelet transforms are classified in two different categories: the continuous wavelet transforms (CWT) and the discrete wavelet transforms (DWT).

Continues wavelet transform (CWT) is defined as the sum over all time of the signal multiplied by scaled, shifted versions of the wavelet function $\Psi$ [6] and [8].

$$
\mathrm{C}(\text { Scale, Position })=\int \mathrm{f}(\mathrm{t}) \Psi(\text { Scale }, \text { Position }, \mathrm{t}) \mathrm{dt}
$$

The results of the CWT are wavelet coefficients C, which are a function of scale and position. Multiplying each coefficient by the appropriately scaled and shifted wavelet yields the constituent wavelets of the original signal $f(t)$ [6] and [8].

The DWT is defined by the following equation [6] and [8].

$$
W(j, k)=\sum_{j} \sum_{k} x(k) e^{-\frac{j}{2}} \Psi\left(2^{-j} n-k\right)
$$

Where $\Psi(t)$ is a time function with finite energy and fast decay called the mother wavelet.

\section{EXPERIMENT}

The ECG signal, consisting of many data points, can be compressed into a few parameters. These parameters characterize the behavior of the ECG signal. In order to extract useful informati on from the ECG signal, the raw ECG signal should be processed. ECG signal processing can be divided into two stages by functionality: Preprocessing and Feature Extraction. The purpose of the feature extraction process is to select and retain relevant information from original signal, using Discrete Wavelet Transform. The preprocessing stage removes or suppresses noise from the raw ECG signal. We use MIT-BIH Arrythmia Database files which includes the normal sinus beat and 13 types of arrhythmia beats. 255 sample signals from different times of these files have been chosen incidentally to provide needed data. Below table shows utilized files and their classifications. The MIT-BIH arrhythmia database is composed of ECG records from 48 subjects with a sampling frequency of $360 \mathrm{~Hz}$, and each record is 30 minutes long.

UTILIZED FILES AND THEIR CLASSIFICATIONS THAT ARE OBTAINED FROM MIT-BIH DATA BASE [9]

\begin{tabular}{cc}
\hline \hline Class & Records \\
\hline Normal & $100-101-103-112-115-117-121-123-202-220-222-234$ \\
RBBB & $212-118-124$ \\
LBBB & $109-111$ \\
\hline \hline
\end{tabular}


The wavelet analysis of ECG signal is performed using MATLAB software. MATLAB is a high performance; interactive system which allows to solve many technical computing problems. The MATLAB software package is provided with wavelet tool box. It is a collection of functions built on the MATLAB technical computing environment. It provides tools for the analysis and synthesis of signals and images using wavelets and wavelet packets within the MATLAB domain. The wavelet transformation is based on a set of analyzing wavelets allowing the decomposition of ECG signal in a set of coefficients. Each analyzing wavelet has its own time duration, time location and frequency band. The wavelet coefficient resulting from the wavelet transformation corresponds to a measurement of the ECG components in this time segment and frequency band.

\section{RESULTS}

Fig. 5 and 6 shows the original ecg and ecg after wavelet filtering.

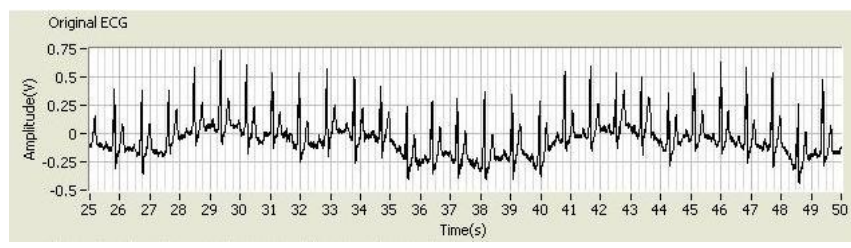

Fig. 5 (Original ECG)

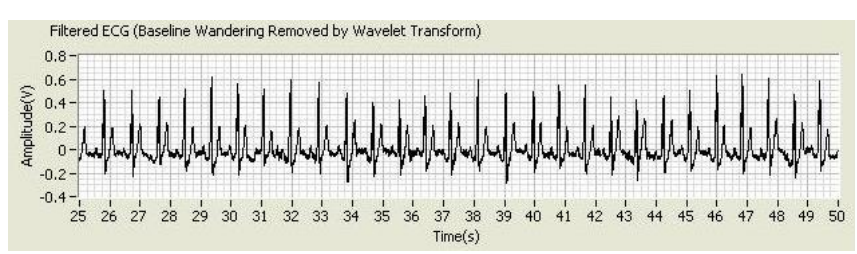

Fig. 5 (ECG after Wavelet Filtering)

There are five classes of heartbeats: the normal beat class ( $\mathrm{N}$ class), the supraventricular ectopic beat class (S class), the ventricular ectopic beat class (V class), the fusion beat class ( $F$ class), and the unknown beat class ( $Q$ class). We reduced the performance variation using dedicated wavelets, as in the ECG morphologies of the subjects. The proposed algorithm utilizes morphological filtering and a continuous wavelet transform with a dedicated wavelet.

The multi-resolution analysis based on the CWT can enhance small differences when the signal is simultaneously observed at the most appropriate scales. Fig. 6 shows the result of the application of the DWT one cycle of a normal ECG. From the figure we can observe that d1 level (frequency ranges of $90-180 \mathrm{~Hz}$ ) emphasize the high frequency content of complex QRS when compared with $\mathrm{P}$ and $\mathrm{T}$ waves. D2 and $\mathrm{d} 3$ levels show clearly that other waves of small frequencies not seen at d1 scale are just appearing.
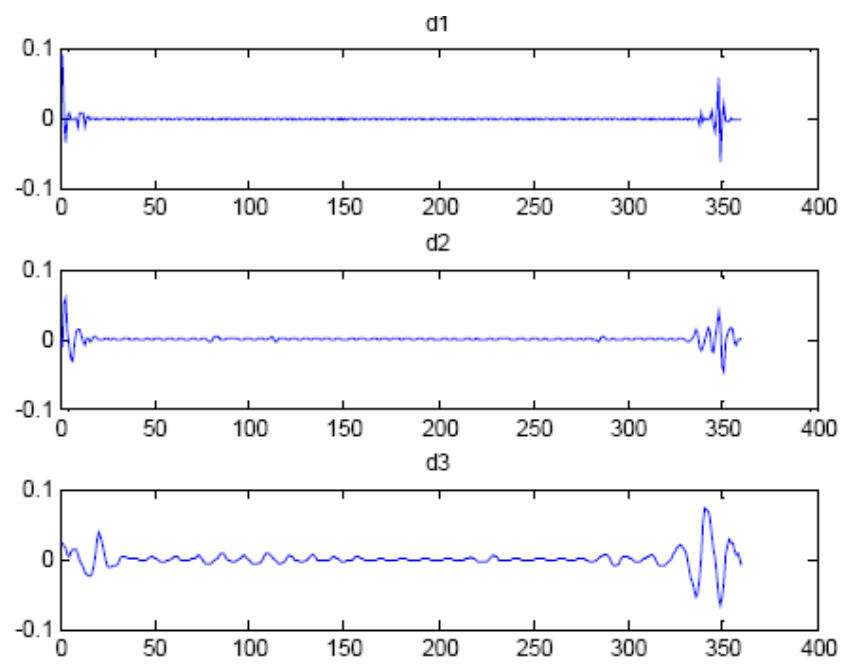

Fig. 6 ECG pulse viewed at scales d1, d2 and d3

\section{Conclusion}

The practical benefit of the wavelet based ECG approach is that T-wave abnormalities can be assessed without the need for T-wave end point identification. The wavelet transformation is a new promising technique in non-invasive electro cardiology providing improved methods for late potential detection. We can observe that the peaks of QRS complexes flatten and $\mathrm{P}$ - and $\mathrm{T}$-waves containing lower frequencies become more visible. At the same time we can also find that details at lower scales constitute the higher frequencies of the signal. The benefit of the wavelet transformation lies in its capacity to highlight the details of the ECG signal with optimal time frequency resolutions. The information about the $\mathrm{R}$ Peak and QRS complex obtained is very useful for ECG Classification, Analysis, Diagnosis, Authentication and Identification performance. The main advantage of this kind of detection is less time consuming for long time ECG signal. It is observed that the no. of QRS waves is less in arrthymia data compared to normal person. The duration between QRS wave to QRS wave is more in arrthymia person.

\section{ACKNOWLEDGEMENTS}

We are thankful to Mr. Vikas Goel, Sr. Project Manager, CDAC, Mohali, Punjab for his valuable guidance and continuous support in making this paper.

\section{REFERENCES}

[1] Martinez, J.P., Almeida, R., Olmos, S., Rocha, A.P., Laguna, P. : A Wavelet-based ECG delineator: Evaluation on standard databases, IEEE Transactions on Biomedical Engineering, vol 51. No. 4, April 2004 , page. $570-561$

[2] Inoue H., A : Noise reduction method for ECG signals Using the dyadic wavelet transform, IEICE Trans. Fundamentals, vol. E81-A, no. 6, 1998 
IOSR Journal of Engineering

Apr. 2012, Vol. 2(4) pp: 632-635

[3] Tikkanen, P. : Characterization and application of analysis methods for ECG and time interval variability data. Faculty of Science, University of Oulu 1999

[4] Almeida, R., Martinez, J.P., Olmos, S., Rocha, A.P., Laguna, P: Automatic Delineationof $\mathrm{T}$ and $\mathrm{P}$ waves using Wavelet-based multiscale approach, International Congress on Computational Bioengineering, madrid, Espana, 2003, pag.243-247

[5] Dubowik, K.: Automated arrhythmia analysis-an expert system for an intensive care unit, Institute of computer science, Technical University of Lodz 2002

[6] L. Cromwell, F.J. Weibell, E.A. Pfeiffer (2005) Biomedical Istrumentation and Measurements, Prentice Hall of India, New Delhi.

[7] J.P. Martinez, R. Almeida, S. Olmos, A.P. Rocha, P. Laguna (2004) IEEE Trans. Biomed. Eng. 51 570-581.

[8] S.Z. Mohmoodabadi, A. Ahmadian, M.D. Abolhasani (2005) ECG feature extraction using daubechies wavelets, Proc. of the fifth IASTED International Conference, Benidorm, Spain.

[9] L. Cromwell, F.J. Weibell, E.A. Pfeiffer (2005) Biomedical Istrumentation and Measurements, Prentice Hall of India, New Delhi. 